\title{
AVALIAÇÃO DA RESISTÊNCIA AO IMPACTO CHARPY DE COMPÓSITOS EPOXÍDICOS REFORÇADOS COM MANTA HÍBRIDA DE MALVA E JUTA*
}

\section{Resumo}

Ygor Macabú de Moraes ${ }^{1}$ Janaina da Silva Vieira² Djalma Souza ${ }^{3}$ Mariana Barcelos ${ }^{1}$ Gabriel Glória ${ }^{1}$ Edson Ribeiro ${ }^{1}$ Sergio Neves Monteiro ${ }^{4}$ Jean Igor Margem ${ }^{5}$

Felipe Perissé Duarte Lopes ${ }^{6}$

Fibras lignocelulósicas vem se tornando alvo de cada vez mais estudos que visam ampliar o aspecto de suas aplicações. Uma de suas aplicações mais importantes é como reforço de compósitos poliméricos na substituição de fibras sintéticas, que não são ambientalmente corretas. Todavia, as fibras ligoncelulósicas com Malva, Juta dentre outras possuem algumas características negativas para sua incorporação em matrizes poliméricas, como por exemplo, o fato dessas não possuírem boa aderência aos polímeros, pelo seu caráter hidrofílico e do caráter hidrofóbico das resinas. Esse trabalho estudou a incorporação de manta hibrida de fibras de malva e juta em compósitos epoxy DGEBA/TETA para testes de impacto Charpy. Os corpos de prova foram feitos sob pressão para que houvesse uma melhora na aderência. $O$ aumento do volume da manta incorporado gerou um aumento da resistência apresentado que pode ser justificado pelo fato da manta atuar como um bloqueador de trincas.

Palavras-chave: Manta de Malva e Juta; Compósitos; Fibras Naturais; Charpy.

\section{EVALUATION OF CHARPY IMPACT RESISTANCE OF EPOXY COMPOSITES REINFORCED WITH HYBRID BLANKET OF MALVA AND JUTE}

\section{Abstract}

Lignocellulosic fibers have become the target of increasingly studies aimed at broadening the aspect of their applications. One the most important applications is as reinforcement of polymeric composites in the substitution of synthetic fibers, that are not environmentally correct. However, cellulosic fibers such as Malva, Juta and others, have some poor characteristics for their incorporation into polymer matrices. For example, the fact they have not a good adhesion because of their hydrophilic characteristic and the hydrophobic character of the resin. This work studies the incorporation of a hybrid blanket of malva and jute in DGEBA / TETA epoxy composites for Charpy impact tests. The specimens were made under pressure to a improvement of adhesion. The increase in volume of incorporated blanket generated an increase in resistance that can be justified by the fact that the blanket act as a blocker cracks.

Keywords: Malva and Jute Blanket; Composites; Natural Fibers; Charpy.

Graduando(a) em Engenharia Metalúrgica e de Materiais, bolsista de iniciação científica, LAMAV, UENF, Campos dos Goytacazes, Rio de Janeiro - Brasil.

2 Mestranda em Engenharia e Ciência dos Materiais, LAMAV, UENF, Campos dos Goytacazes, Rio de Janeiro - Brasil.

3 Engenheiro Metalúrgico, Doutor em Ciência e Engenharia de Materiais, Professor, LAMAV, UENF, Rio de Janeiro, Rio de Janeiro - Brasil. 
4 Engenheiro Metalúrgico, Doutor em Ciência e Engenharia de Materiais, Professor titular, IME, Rio de Janeiro, Rio de Janeiro - Brasil.

5 Engenheiro Metalúrgico, Doutor em Ciência e Engenharia de Materiais, Instituto de Ensino Superiores do Censa (ISECENSA), Campos dos Goytacazes - RJ, Brasil.

6 Engenheiro Metalúrgico, Doutor em Ciências de Materiais, Livre-Docência, LAMAV, UENF, Campos dos Goytacazes, Rio de Janeiro - Brasil. 


\section{INTRODUÇÃO}

Nos últimos anos, o uso de materiais compósitos vem crescendo significativamente e isso se deve a diversos fatores, dentre os quais podemos destacar a sua alta resistência específica, que é a resistência por unidade de massa. A resistência específica de um compósito pode chegar até a $400 \mathrm{MPa} . \mathrm{cm} 3 / \mathrm{g}$ sendo muito maior do que qualquer outro material monolítico como metais, cerâmicas e polímeros. Essa característica faz com que os materiais compósitos sejam praticamente ideias para onde se quer alta resistência com baixo peso que é o caso da indústria aeroespacial e automobilística de ponta. Portanto, os materiais compósitos apresentam uma boa resistência aliado a um baixo peso.

Todavia, o uso de compósitos que utilizam fibras sintéticas como reforço acaba sendo prejudicial ao meio ambiente, pois as fibras sintéticas emitem muito $\mathrm{CO}_{2}$ para serem produzidas e, além disso, não podem ser recicladas e nem incineradas [1]. Surge então, como alternativa a aplicação de fibras naturais no lugar das sintéticas. As fibras naturais lignocelulósicas vêm sendo alvo de várias pesquisas [2-6], pois são ambientalmente corretas e também possui custo de produção menor, já que na maioria do caso são materiais de descarte de outros processos. Determinadas fibras naturais já vem sendo empregadas industrialmente no setor automotivo na produção de peças de acabamento interno [7-8].

Entretanto, as fibras naturais também possuem pontos negativos, como por exemplo o fato de serem hidrofílicas enquanto as matrizes poliméricas serem hidrofóbicas, com isso tornasse mais difícil haver uma boa interação da manta com a resina. $O$ objetivo desse trabalho é analisar a resistência ao impacto charpy da manta híbrida das fibras de Malva e Juta.

\section{MATERIAIS E MÉTODOS}

Os materiais utilizados nesse trabalho foram a reina comercial epoxi DGEBA/TETA com phr13, proveniente da empresa Resinpoxy e a manta híbrida de Malva e Juta foi adquirida da empresa PERMANTEC. Na Fig. 1 podemos ver as fibras de Malva (a) e de Juta (b) e a manta hibrida de Malva e Juta (c).

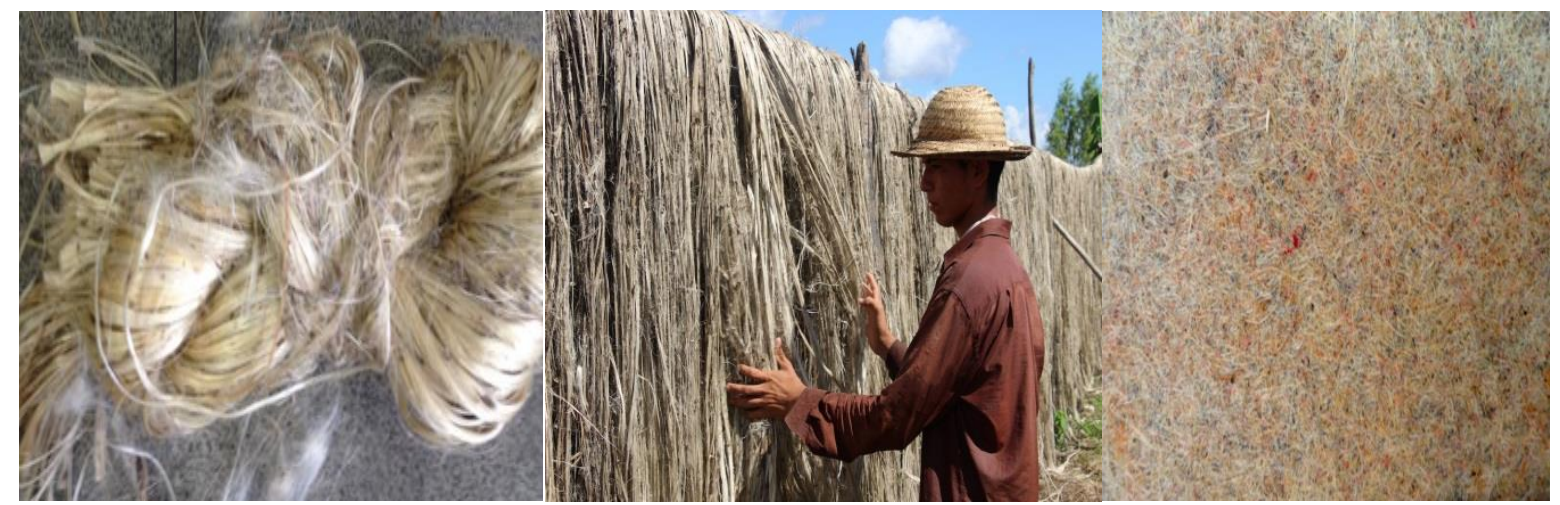

Figura 1. Fibras de (a)Malva, (b) Juta e (c) a manta hibrida de Malva e Juta.

A densidade da manta foi analisada pelo processo de picnometria onde foi encontrado o valor $1,05 \mathrm{~g} / \mathrm{cm}^{3}$ para sua densidade. Para confecção dos copors de prova, a manta foi inicialmente seca em estufa a $60 \mathrm{C}^{\circ}$ para que fosse retirada parte de sua umidade residual. Após a pré secagem, a manta foi cortada com o auxílio de um estilete e então posta em um molde metálico juntamente com a resina, já com o 
catalizador e posta sob pressão em uma prensa hidráulica para cura por 24 horas a temperatura ambiente. Foi confeccionados corpos de prova com percentual de manta variando entre 0 e $30 \%$ em volume.

Depois de retiradas do molde, as amostras foram polidas para que essas tivessem uma melhor qualidade superficial e então foram ensaiadas em uma máquina de impacto Pantec, Figura 2, em ambiente controlado de acordo com a norma ASTM D256.

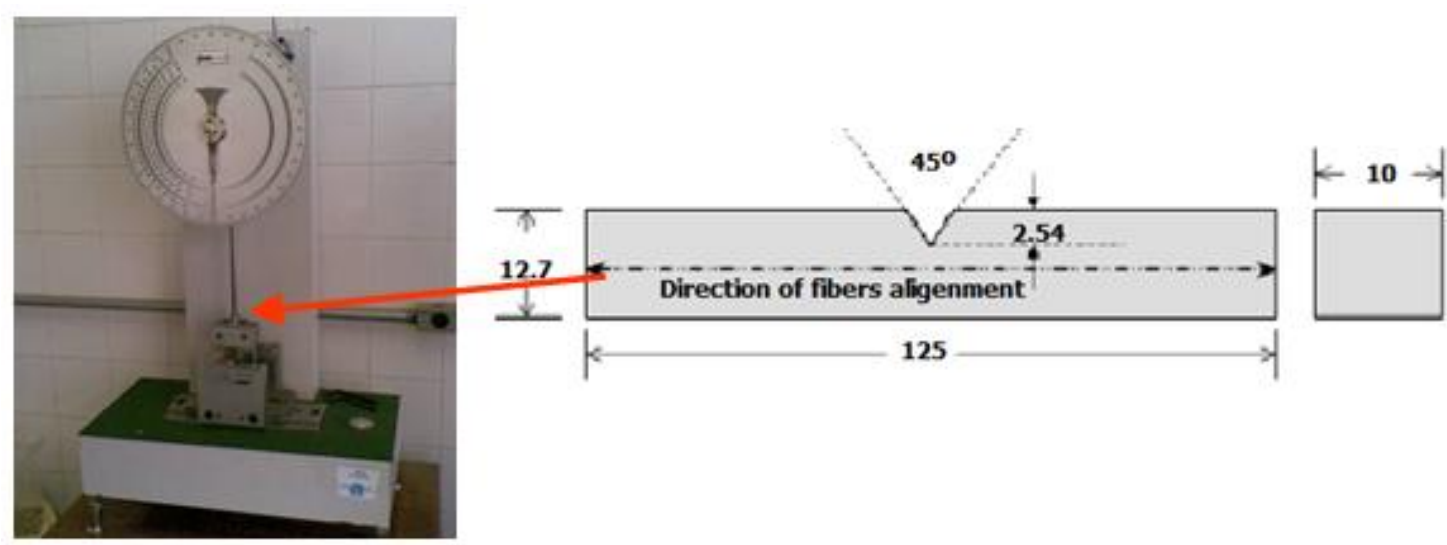

Figura 2. Máquina de impacto Pantec e modelo do corpo de prova.

\section{RESULTADOS E DISCUSSÃO}

A Tabela 1 mostra os resultados do ensaio de impacto Charpy/lzod dos compósitos de epoxy de manta hibrida de Malva de Juta.

Tabela 1. Variação da energia de impacto charpy com o volume de manta hibrida de malva/juta incorporada

\begin{tabular}{c|c}
\hline $\begin{array}{c}\text { Percentua de } \\
\text { Fibra (\%) }\end{array}$ & $\begin{array}{c}\text { Energia de Impacto } \\
\text { Charpy (J/m) }\end{array}$ \\
\hline 0 & $22,9 \pm 9,7$ \\
\hline 10 & $60,5 \pm 14,7$ \\
\hline 20 & $120,5 \pm 24,5$ \\
\hline 30 & $168,6 \pm 31,6$ \\
\hline
\end{tabular}

Baseado nos resultados da Tabela 1 foi plotado os gráficos da variação da energia de impacto absorvida pelos corpos de prova com o percentual de manta incorporada nos compósitos, Fig. 3. 


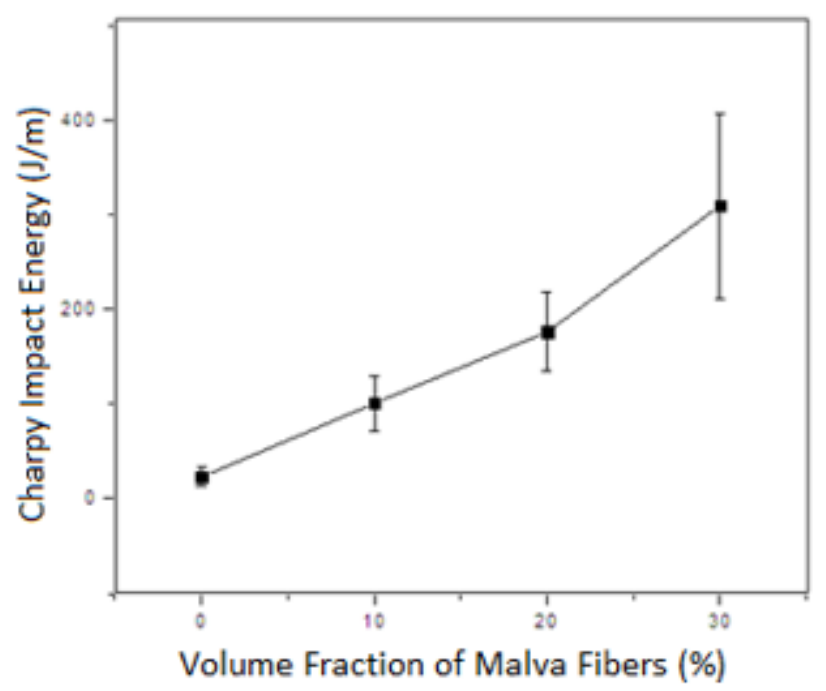

Figura 3. Variação da energia de impacto charpy absorvida pelos compósitos incorporados com a variação do percentual da manta.

A Tabela 2 mostra a energia de impacto chapry de outras fibras incorporadas a resina epoxy DGEBA/TETA [9].

Tabela 2. Variação da energia de impacto Charpy de outras fibras naturais

\begin{tabular}{c|c}
$\begin{array}{c}\text { Compósitos Epoxy } \\
\text { com 30\% de Fibra }\end{array}$ & $\begin{array}{c}\text { Energia de Impacto } \\
(\mathbf{J} / \mathbf{M})\end{array}$ \\
\hline Malva & 310,2 \\
\hline Ramie & 211,7 \\
\hline Coco & 174,7 \\
\hline Curaua & 103,2 \\
\hline
\end{tabular}

Os valores apresentados na Fig. 3 são consistentes com os resultados relatados na literatura [7-10]. Ou seja, os compósitos absorvem quantidades de energia relativamente maiores, levando a um aumento da resistência ao impacto com o aumento de incorporação de mantas em volume até $30 \%$.

O fato das mantas permanecerem intactas após o impacto, como mostrada para os compósitos na Fig. 4, é uma indicação de que as trincas se propagaram ao longo da interface tecido / matriz, causando a separação da manta da resina epóxi, mas não a completa ruptura da manta. Esse efeito aumenta a trajetória das trincas através do composto, criando uma maior energia de impacto. 


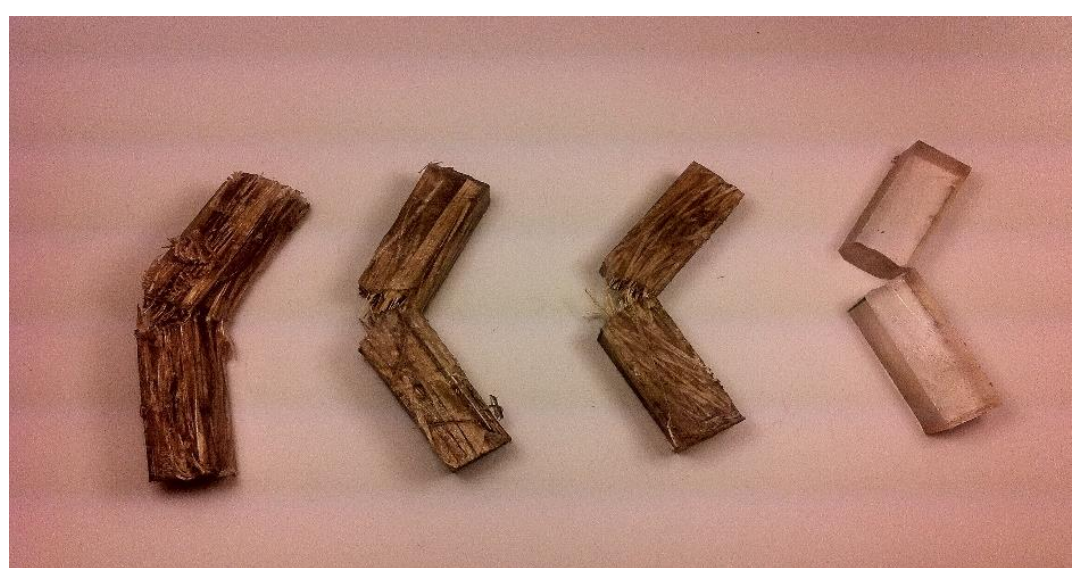

Figura 4. Macrografia das amostras de compósitos epoxy reforçados com manta de malva e juta.

\section{CONCLUSÃO}

- O aumento do percentual de mantas de fibras gera um aumento da resistência ao impacto.

- Grande parte desse aumento é, aparentemente, devido à baixa resistência de cisalhamento interfacial do tecido / matriz epoxi tensionada. Isso resulta em maior energia absorvida como resultado da propagação de fissuras longitudinais ao longo da interface, o que leva a maiores áreas de ruptura em comparação com uma fratura transversal.

\section{REFERÊNCIAS}

[1] Wambua P, Ivens I, Verpoest I. Natural Fibers: Can They Replace Glass And Fibre Reinforced Plastics?, Composites Science and Technology, 63 (2003) 1259-1264.

[2] Nabi Sahed D, Jog JP. Natural Fiber Polymer Composites: A Review, Advances in Polymer Technol., 18 (1999), 221-274

[3] Mohanty AK, Misra M, Hinrichsen G. Biofibers, Biodegradable Polymers And Biocomposites: An Overview, Macromolecular Mat. And Engineering, 276/277 (2000), 1-24

[4] Eichhorn SJ, Baillie CA, Zafeiropoulos N, Mwakambo LY, Ansell MP, Dufresne A. Review Of Current International Research Into Cellulosic Fibres And Composites, J. Mater. Science, 36 (2001) 2107-2113

[5] Monteiro SN, Lopes FPD, Ferreira AS, Nascimento DCO. Natural Fiber Polymer Matrix Composites: Cheaper, Tougher And Environmentally Friendly. JOM, 61(1) (2009) 17-22.

[6] Mohanty AK, Misra M, Drzal LT. Sustainable Biocomposites From Renewable Resources: Opportunities And Challenges In The Green Material World", J. Polym. Environ., 10 (2002), 19-26

[7] Monteiro SN, Ferreira AS, Lopes FPD. A Comparative Study Of Curaua Fiber Reinforced Epoxy Matrix Composites As Building Materials, Proceedings of the Global Symposium on Recycling, Waste Treatment and Clean Technology REWAS2008, (Cancun, Mexico, October 2008) 1653-1658.

[8] Monteiro SN, Satyanarayana KG, Lopes FPD. High strength natural fibers for improved polymer matrix, Mat. Sci. Forum 638-642, 2010, 961-966.

[9] Monteiro SN, Margem FM, Santos Jr LFL. Ensaios De Impacto Izod Em Compósitos Poliméricos Reforçados Com Fibras De Rami. In: $18^{\circ}$ Congresso 
Brasileiro de Engenharia e Ciencia dos Materiais, CBECIMAT 2008, Porto de Galinhas, PE, November, 2008, p. 1-12.

[10] Monteiro SN, Ferreira AS, Lopes FPD. Izod Impact Energy Of Polyester Matrix Composites Reinforced With Aligned Curaua Fibers. In: Mineral, Metals \& Materials Characterization Symposium - TMS Conference, San Francisco, EUA, March 2009. p. 1-8.

[11] Inácio WP, Lopes FPD, Monteiro SN. Charpy Toughness Behavior Of Continuous Sisal Fiber Reinforced Polyester Matrix Composites, In: Mineral, Metals \& Materials Characterization symposium - TMS conference, Seattle, EUA, March, p. 1-8, 2010. 